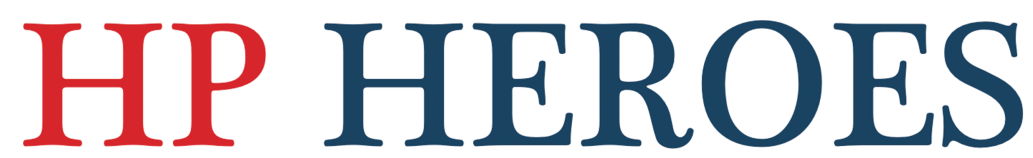

CANADIAN SOCIETY OF INTERNAL MEDICINE

By Bert Govig for the CSIM Health Promotion Committee

The CSIM Health Promotion Committee promotes knowledge, culture, skills, and the practice of Health Promotion by physicians, patients, communities, and health care systems. The HP Heroes series highlights inspirational work in the diverse field of health promotion. Submitted: July 23, 2019. Published: August 31, 2019. DOI: 10.22374/cjgim.v14i3.388

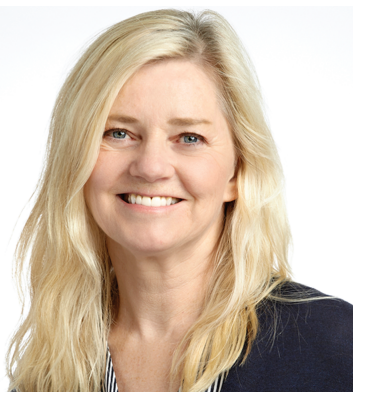

\title{
Dr. Jane Lemaire
}

Dr. Jane Lemaire is a physician of many interests, talents, and accolades, as illustrated by a few morsels, plucked from her c.v.

- Clinical Professor - Division of GIM

- Vice-Chair - Physician Wellness and Vitality, Department of Medicine at the Cumming School of Medicine, University of Calgary.

- 2005 Osler award winner (this is a career achievement award and the highest honour that the CSIM can bestow on one of its members).

- 2016 CMA Misericordia award for contributions to physician wellness.

She and her research colleagues have passionately advocated for recognition of physician wellness as a quality indicator of healthcare systems, and shared responsibility for physician wellness that lies with individual physicians, the medical profession, and healthcare systems.

\section{What sparked your interest in physician wellness?}

I feel like I have always known that physicians are at risk of being unwell by virtue of the very important and intense work that we do. The combinations of the generational and gender shifts in medicine, and the increasing complexity of healthcare delivery signalled that our profession was evolving to be more demanding than ever before. I started on staff in the early 1990 s as a female, mother, spouse, academic physician, and consultant in General Internal Medicine. It was apparent to me that I would not survive in the profession unless there was advocacy for wellness, and some means to mitigate the profession-related work hazards. I think I kind of "walked the walk" and "talked the talk" early on (likely to the dismay of some of my colleagues!), taking the occasional afternoon off to regroup, making time for family and physical fitness, trying to be a good citizen and pull my weight, but setting boundaries for myself and advocating for a sustainable work environment for all. Then, in 2004, through discussions with our visionary Department of Medicine (DOM) Chair at the time - Dr. John Conly - we created the formal position of vice-chair of Physician Wellness and Vitality.

\section{Describe your current wellness-related work.}

I have been extremely fortunate in that the physician wellness work has been supported by so many over the years. Even though I have several formal titles, the work more or less all overlaps. Our wellness team strived to find the balance between conducting research to further the science and then translating that knowledge into action. Since 2004, the DOM has allowed me to use my position for physician wellness work that extends beyond our department borders - locally, provincially, nationally, and internationally. This has led to culture change and behavior change. I believe that Alberta is ahead of the curve in physician wellness. There are many reasons for this. We have an amazing Alberta Medical Association provincial health program - the Physician and Family Support Program. There is the ongoing work of so many individuals and groups working towards wellness. So, although I have the formal positions as outlined in my bio, I feel like I have been part of this wonderful journey where physicians across the province have taken the "leap" and committed to engage, learn, self-reflect, and consider change based on the science of physician wellness. There is a lot of 
work to do. But we have engagement and empowerment at the grassroots level, and support from leadership within our formal healthcare systems - they understand the cost of unwell physicians to the system and to patient care, and they value physicians as a human resource.

Jane, in addition to many other hats, you are the Director of Wellness at the Office of Professionalism, Equity, and Diversity, as well as the Wellness Lead at the W21C Research and Innovation Center, Cumming School of Medicine, University of Calgary. That group has done some interesting research. Could you share some of that with us?

The W21C Wellness team has studied determinants of physician wellbeing, explored workplace nutrition on physician wellness, and studied the influence of coping strategies and personalities on physician wellness. Recent research explores how contextual factors within the complex healthcare work environment are linked to physician wellness, and patients' views on physician wellness and how it links to patient care. Since 2018, with support from provincial stakeholders in healthcare, you and the wellness team created the Well Doc Alberta Initiative, a pan-provincial approach to physician wellness, now funded for three years thanks to the combined support of the Canadian Medical Association, Scotiabank, and MD Financial Management.

\section{Can you tell us a bit more about Well Doc Alberta?}

This initiative is meant to fill in the gap - we have a wonderful physician health program - now we need to focus on education and prevention. Our vision is "Shaping the future of physician wellness, together". We stand as an independent initiative, with all of the healthcare organizations and patients within Alberta as our stakeholders. We hope to harness the experience of so many involved in wellness work across Alberta using a cooperative, collaborative model. Well Doc Alberta's purpose is to foster culture change, and ultimately drive systems-level change in support of physician wellness through prevention. The science of physician wellness can empower people and organizations to decrease the risk to physicians.

Jane, you are an obvious and natural leader for this cause - as you mention, you walked the wellness walk from early on in your practice. That said, moving from a clinical practice to a field that involved a lot of social science research and policy-oriented work must have been disorienting. How did that happen?

When I first came to Calgary, it was to pursue a fellowship in Critical Care and Pulmonary Medicine. But they needed GIM consultants and offered me a position. Not exactly what I had planned, but it seemed like a great opportunity to jump right into my career and help develop the division. In retrospect it was perfect! We always think we should have control over every little part of our lives, but sometimes serendipity is the greatest opportunity. Similarly, after over a decade of medical education as the focus of my career, Dr. Conly asked me to consider the vice-chair position in physician wellness. I was terrified as I had no idea what I would do. So, I told John that he had to give me a whole year to figure it out. He did, and here I am 15 years later. I've worked with the most incredible people, made a difference, learned so much (on sabbatical one year I audited a graduate level class on qualitative research), and established relationships with world experts in physician wellness (who are now great friends as well as colleagues). The generous involvement of physicians across Alberta in our research provided the raw data for science. I have heard the stories of hundreds of physicians and observed many in their work. The narratives are both sobering and inspiring.

\section{What are 3 things outside medicine that make your heart go pitter-patter?}

- I love, love, love to downhill ski with my husband Chris. That is why I live in Calgary. I have literally cried tears of joy and wonder as I whooshed through bottomless powder for 2500

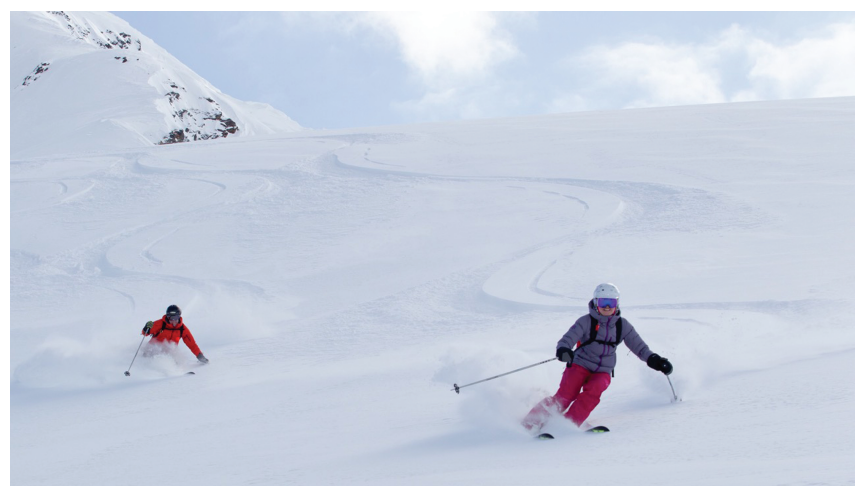

Skiing Blue River. 
vertical feet. Grateful to mother nature, for my health, for living in a peaceful, just country, for still being able to pursue athletic sports at age 62 , and for being the first one down!

- My older daughter decided we should have some motherdaughter bonding a few years back. We started to sport climb together, mostly indoor at a climbing center, but with the occasional foray into the outdoors. Once I overcame my fear of heights, I was able to delight in this new, very technical, meditative, and physical sport. The other climbers encourage and support me, and the time with my coach/daughter is magnificent. My husband Chris also joined in.

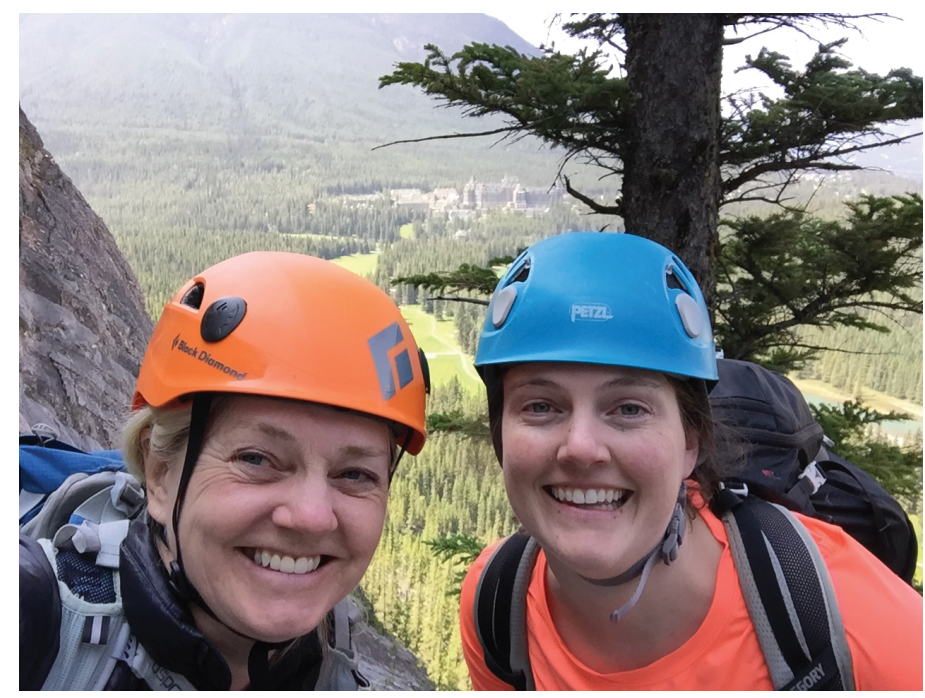

Jane and her daughter Garielle Brown in climbing helmets.

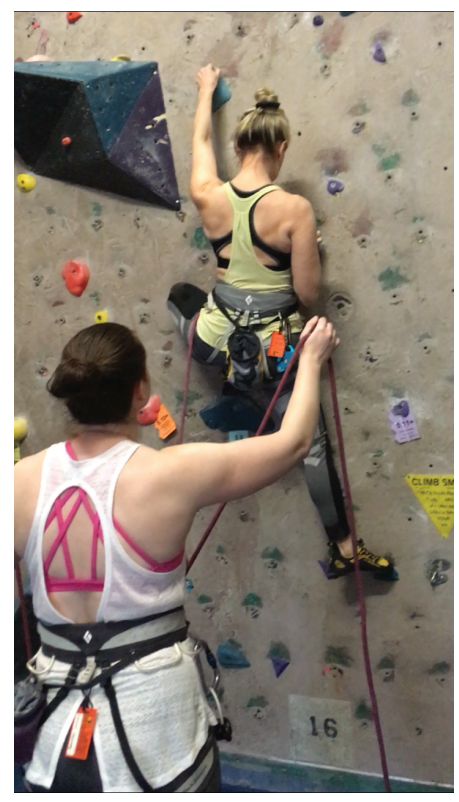

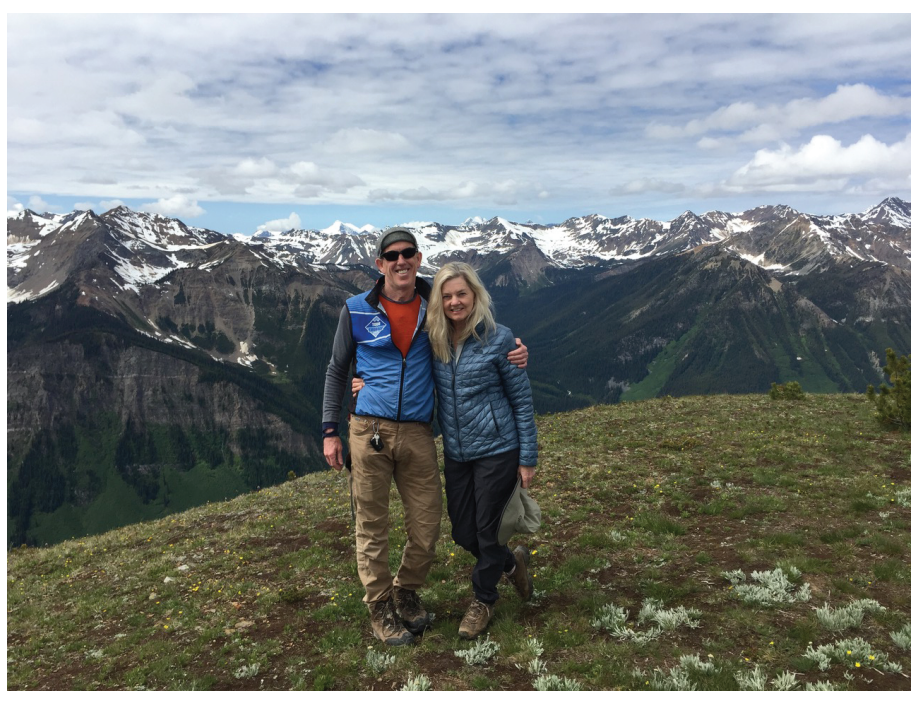

\section{Jane and husband Chris.}

- One of my favorite things to do is having face time conversations with my younger daughter Pier Brown who is studying at the University of Victoria. Through our conversations, she has educated me immensely around her diverse areas of interest that include such topics as social activism, inequity, history, and on and on. Visits to Victoria are spent on field trips beachcombing with her teaching me marine biology. Smart kids ... and so much more for me to learn.

In short, then your heartstrings relate to your relationships and particularly family. I love the answer and feel like there's a nice wellness lesson for all of us here.

\section{If you had a magic wand and could impose a single condition or behaviour on all of the population to improve overall health - what would it be?}

Kindness....

\section{What is your secret Canadian vacation that you would urge Canadians to spoil themselves with?}

Go into the Canadian Arctic - before it is changed forever. Adventure Canada is a Canadian company, a small expedition cruise, 150 people, with a faculty of geologists, marine biologists, ornithologists, arctic historians, anthropologists, Inuit culturalists, etc ... fantastic trip.

Where can people learn more about the projects that matter most to you?

www.welldocalberta.org

Jane the climber. 